\title{
Modulation of matrix metalloproteinases MMP-2 and MMP-9 activity by hydrofiber-foam hybrid dressing - relevant support in the treatment of chronic wounds
}

\author{
ALICJA KREJNER, TOMASZ GRZELA \\ Department of Histology and Embryology, Medical University of Warsaw
}

\begin{abstract}
Success in chronic wound therapy requires careful selection of appropriate dressing, which enables effective management of wound exudate. According to current knowledge, exudate may contain large quantities of proteases, including matrix metalloproteinases, MMP-2 and MMP-9, which are responsible for delay in wound healing. Therefore, neutralization of MMPs may be beneficial for treatment efficacy. The aim of the study was to test whether AQUACEL Foam, a novel, technologically advanced hydrofiber-foam hybrid dressing (HFHD), may interfere with proteolytic activity of MMPs in vitro.

The assessment included in vitro tests of liquid retention and measurement of human recombinant $M M P-2$ and MMP-9 activity. The MMPs activity was measured before and after their interaction with $H F H D$, using a fluorescent gelatinase assay kit and Real-Time PCR device. The in vitro tests have shown that the hydrofiber layer of HFHD revealed significant potential to decrease the activity of MMPs in the experimental system. The mentioned modulatory properties of AQUACEL Foam may contribute to a composed mechanism of its beneficial action. Furthermore, our finding may explain clinical effectiveness of HFHD observed in clinical settings.
\end{abstract}

Key words: chronic wound, hybrid dressing, hydrofiber, matrix metalloproteinase, MMP.

(Cent Eur J Immunol 2015; 40 (3): 391-394)

\section{Introduction}

Chronic wounds, including venous leg ulcers, constitute a serious problem in many developed countries, with a significant load for the health budget [1]. Due to a pain and odorous exudate they impair the patient's business and social activity [2]. Therefore, their effective management is in focus of interest of health care systems. According to current knowledge, success in chronic wound therapy, in addition to appropriate causative treatment (e.g. providing sufficient blood perfusion, control of infection), requires proper local treatment. It is well known that moist wound environment is essential for proper wound healing. Appropriate moisture is required for cell migration and cytokines action. A dry wound bed promotes eschar formation, which impedes wound healing and contraction [3, 4]. On the other hand, chronic wound exudate contains high levels of proteases which are responsible for peri-wound skin maceration and, finally, delay in the wound healing process. It has been found that excessive exudate may decrease proliferation of fibroblasts, keratinocytes and endothelial cells $[5,6]$. Hence, in order to deal with key aspects of chronic wounds pathophysiology, various dressings were developed. Unfortunately, there is no one universal dressing, which could be used in each wound, or at any stage of wound healing. Nevertheless, in recent years, several technologically advanced products have been developed. They combine different materials and their unique properties and, therefore, they may be defined as "hybrid" dressings.

One of such modern dressings has recently been developed by ConvaTec. It consists of three layers. The innermost wound contact layer is composed of gel-forming sodium carboxymethylcellulose (NaCMC fiber, also known as hydrofiber). The NaCMC fibers in contact with wound exudate form a gel-like structure, which retains some fluid to keep wound environment moist that is necessary for successful wound healing. Noteworthy, such a gel-forming layer may be removed without damage to the regenerating immature tissue [7]. As the hydrofiber layer reaches its capacity, the excess fluid moves horizontally into the upper hydrophilic polyurethane foam pad. Thus, in addition to maintaining moist environment, hydrofiber-foam hy-

Correspondence: Tomasz Grzela, Department of Histology and Embryology, Medical University of Warsaw, e-mail: tomekgrzela@gmail.com 
brid dressing (HFHD) absorbs and immobilizes excessive amounts of wound exudate, and thus it may reduce the risk of maceration. The outermost, waterproof layer protects against uncontrolled move of exudate from the HFHD to bandages and migration of bacteria in the opposite direction $[8,9]$. Due to mentioned properties, HFHD is dedicated to treat wounds with moderate or large quantities of the wound fluid. Moreover, it is supposed to neutralize the detrimental effect of some biologically active molecules, such as matrix metalloproteinases (MMPs).

Matrix metalloproteinases belong to a family of zinc-dependent proteases and are responsible for cleavage of extracellular matrix (ECM) components, e.g. collagen, elastin and laminin [10]. It has been shown that MMPs play a crucial role in the tissue remodeling and wound healing process [11]. However, an excessive activity or imbalance between MMPs and their endogenous inhibitors (tissue inhibitors of metalloproteinases, TIMPs) may lead to extensive destruction of tissue $[12,13]$, thus augmenting the chronic inflammatory reaction in the wound bed. Numerous studies have confirmed a relationship between the delayed healing process and increased levels of MMPs (mainly MMP-2 and MMP-9) in wound exudate [14]. Based on these observations it is supposed that modulation of MMPs activity may be beneficial to improve the wound treatment efficacy. Therefore, we have tested using an in vitro model whether reported clinical effectiveness of HFHD may depend on its interference with MMPs activity.

\section{Material and methods}

\section{In vitro liquid retention tests}

Selected properties of HFHD (AQUACEL Foam, ConvaTec, Deeside, UK) were analyzed using samples of dressing, $1 \mathrm{~cm}^{2}$ each, and human recombinant MMP2 and MMP-9 (R\&D Systems, Minneapolis, MN, USA). Hydrofiber-foam hybrid dressing patches were placed into 6-well polystyrene culture plates (Corning Inc., Corning, NY, USA). Then, $300 \mu$ l of MMP-2 or -9 solution (500 ng/ $\mathrm{ml}$ ), preactivated with $1 \mathrm{mM} 4$-aminophenylmercuric acetate (APMA; Sigma, St. Louis, MO, USA), were applied to soak the dressing sample and incubated for 1 hour, at $37^{\circ} \mathrm{C}$. After incubation, foam pad and gel-forming hydrofiber layers were split and centrifuged separately at 13,500 rpm $(12,000 \times \mathrm{g})$ for $5 \mathrm{~min}$. Supernatants from the foam layer were collected and stored at $4{ }^{\circ} \mathrm{C}$, until further testing. In the case of the hydrofiber layer, an additional washing step with $300 \mu \mathrm{l}$ of PBS was performed for $1 \mathrm{~h}$, at $37^{\circ} \mathrm{C}$, followed by centrifugation at $13,500 \mathrm{rpm}$, for $5 \mathrm{~min}$.

\section{Modulation of MMPs activity}

Matrix metalloproteinases activity in supernatants and eluates was determined using the fluorescent EnzChek
Gelatinase/Collagenase Assay Kit (Invitrogen, Carlsbad, CA, USA), according to the protocol described in detail elsewhere [15]. The fluorescence of the samples was monitored using the ABI PRISM 7500 Real-Time PCR device (Applied Biosystems, Foster City, CA, USA). The enzyme activity was calculated based on the standard curve created for serial dilutions of MMP-2 or -9 , preactivated with APMA.

Furthermore, $20 \mu \mathrm{l}$ of reaction mixtures, containing fluorescein-conjugated gelatin, APMA-preactivated MMP-2 or -9 , and hydrofiber samples (approx. $1 \mathrm{~mm}^{3}$ each) were incubated in 96-well PCR optical plates (Axygen Scientific Inc., Union City, CA, USA) for $1 \mathrm{~h}$, at $37^{\circ} \mathrm{C}$. Afterwards, fluorescence of samples was measured as described above. The fluorescent substrate with MMP- 2 or -9 served as the positive control for enzyme activity, whereas samples containing substrate alone or substrate with hydrofiber specimens, without MMPs, were used as the background control. Samples of APMA-preactivated MMP-2 or -9, incubated with the fluorescent substrate in the presence of $1 \mathrm{mM}$ phenanthroline - a broad spectrum metalloproteinase inhibitor (Invitrogen, Carlsbad, CA, USA), were used as the inhibition control. The in vitro experiments were repeated twice, each sample was run in duplicates.

\section{Results}

The in vitro experiments have shown that the tested dressing (HFHD) displayed liquid-binding capacity of about $0.3 \mathrm{ml}$ per $\mathrm{cm}^{2}$. Approximately $30-35 \%$ of that volume were loosely immobilized in the foam pad and were easily retrievable by high-speed centrifugation. The remaining $65-70 \%$ were absorbed by the gel-forming hydrofiber layer so firmly that even centrifugation at $13,500 \mathrm{rpm}(12,000 \times \mathrm{g})$ was ineffective to get any supernatant from samples of the hydrated/saturated hydrofiber layer. Therefore, saturated hydrofiber samples were subjected to an additional washing step with $0.3 \mathrm{ml}$ of PBS. After centrifugation, eluates were collected and used for further analysis.

The analysis of supernatants and eluate samples was performed using fluorescent EnzChek Gelatinase/Collagenase Assay. It has been shown that HFHD components revealed various ability to influence the in vitro gelatinolytic MMPs activity. In supernatants obtained from foam pads, the mean MMP-2 or -9 activity, when compared to APMA-preactivated control, was reduced to $53 \%$ and $68 \%$ of the initial value, respectively. In eluate samples, obtained from the saturated hydrofiber layer, mean MMP-2 or -9 activity corresponded to $7 \%$ and $5 \%$ of the positive control, respectively (Fig. 1).

The incubation of APMA-preactivated MMP-2 or -9 with fluorescein-labeled gelatin and fresh hydrofiber samples, added directly to the reaction mixture, resulted in a significant decrease of initial MMP-2 or -9 activity, respectively, to $32 \%$ and $37 \%$ of the control (Fig. 2). 


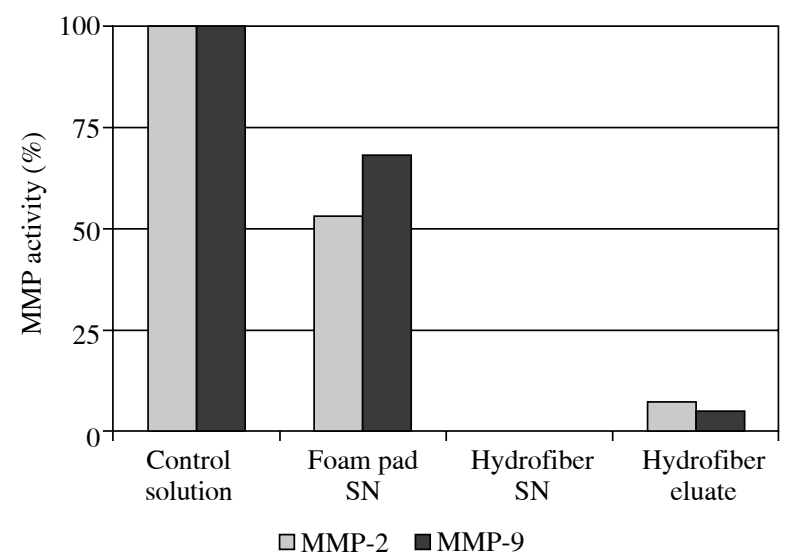

Fig. 1. Activity of MMP-2 and MMP-9 solutions (shown in \%) measured before (control solution) and after incubation with AQUACEL Foam dressing (Foam pad SN and Hydrofiber SN, respectively), followed by centrifugation. Only minute quantities of MMPs were eluted with PBS from the hydrofiber layer, which did not release any MMPs to the SN

\section{Discussion}

The results of our study have shown for the first time that AQUACEL Foam, a novel hydrofiber-foam hybrid dressing, possesses potential to reduce activity of MMPs. Thus, in addition to relatively high absorption capacity of the analyzed product, the observed modulatory properties may explain clinical effectiveness of this dressing, reported in previous studies $[16,17]$. The main action of HFHD, postulated so far, was the maintaining of optimal environment for tissue regeneration and better management of the excessive wound fluid. It resulted in a lesser need of frequent dressing change, but also in reduced irritation of the peri-wound skin. The latter might be due to slighter skin maceration, however, based on our experimental data, this effect may also be related to hydrofiber-mediated modulation of MMPs activity.

The key role of MMPs has been considered to be essential in many aspects of wound healing, including wound debridement and long-term tissue remodeling [18]. However, an unrestrained production and hyperactivation of MMPs contributes to excessive ECM degradation and results in impaired healing $[11,19]$. Several studies have proven a direct relationship between the increased activity of MMP-2 and MMP-9 in the chronic wound fluid, and delayed healing of chronic venous ulcers [14, 20, 21]. Moreover, some authors have demonstrated that intentional MMP inhibition may be crucial for effective management of chronic wounds [15, 22].

The mechanism of modulation of MMPs activity by AQUACEL Foam dressing remains unclear. Our study revealed that the hydrofiber layer of HFHD was able to

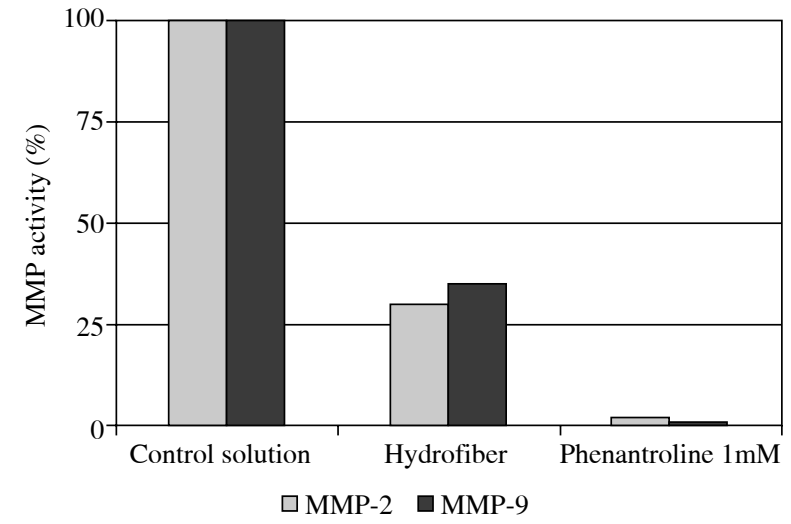

Fig. 2. Activity of MMP-2 and MMP-9 solutions (shown in \%) measured before (control solution) and after addition of hydrofiber sample (Hydrofiber) or Phenanthroline $(1 \mathrm{mM})$ directly to the reaction mixture

absorb and immobilize certain volume of liquid containing MMP-2 or MMP-9. Moreover, the activity of MMPs in liquid, which passed through the hydrofiber and was collected from the upper foam layer, was significantly reduced, as compared to their initial (100\% control) activity. Possibly, the observed reduction in MMPs activity seems to be due to a simple lock of active MMPs in a gel-like structure of the hydrofiber layer. Thus, since immobilized in the hydrofiber layer, MMPs have no access to their matrix substrates of the wound bed. However, in our second experimental system, we have used a liquid substrate, which could easily access MMPs, even immobilized in hydrofiber samples, present in the reaction mixture. Interestingly, also in that system MMPs activity was significantly reduced. Therefore, some other mechanisms, including direct inhibition of MMPs by carboxymethylcellulose, or chelating of $\mathrm{Zn}^{2+}$ or $\mathrm{Ca}^{2+}$ ions, which are necessary for MMPs activity, cannot be excluded.

In summary, AQUACEL Foam, a novel hydrofiber-foam hybrid dressing, may offer relevant support in wound healing. An effective management of wound exudate, combined with immobilization/neutralization of detrimental MMPs allows for protection of the wound tissue and peri-wound skin from proteolytic destruction, but also enables reduction in dressing change frequency. Since the effectiveness and comfort of treatment are much improved, the concept of hybrid dressings seems to be especially promising and should be further developed.

The authors declare no conflict of interest. 


\section{References}

1. Mekkes JR, Loots MA, Van Der Wal AC, Bos JD (2003): Causes, investigation and treatment of leg ulceration. $\mathrm{Br} \mathrm{J}$ Dermatol 148: 388-401.

2. Bradbury AW (2010): Epidemiology and aetiology of C4-6 disease. Phlebology 25 Suppl 1: 2-8.

3. Leaper DJ, Schultz G, Carville K, et al. (2012): Extending the TIME concept: what have we learned in the past 10 years? Int Wound J 9 Suppl 2: 1-19.

4. Okan D, Woo K, Ayello EA, Sibbald G (2007): The role of moisture balance in wound healing. Adv Skin Wound Care 20: 39-53.

5. Stanley AC, Park HY, Phillips TJ, et al. (1997): Reduced growth of dermal fibroblasts from chronic venous ulcers can be stimulated with growth factors. J Vasc Surg 26: 994-1001.

6. O'Toole EA, Marinkovich MP, Hoeffler WK, et al. (1997): Laminin-5 inhibits human keratinocytes migration. Exp Cell Res 233: 330-339.

7. Waring MJ, Parsons D (2001): Physico-chemical characterisation of carboxymethylated spun cellulose fibres. Biomaterials 22: 903-912.

8. Blome-Eberwein S, Johnson RM, Miller SF, et al. (2010): Hydrofiber dressing with silver for the management of split-thickness donor sites: a randomized evaluation of two protocols of care. Burns 36: 665-672.

9. Walker M, Hobot JA, Newman GR, Bowler PG (2003): Scanning electron microscopic examination of bacterial immobilisation in a carboxymethyl cellulose (AQUACEL®) and alginate dressings. Biomaterials 24: 883-890.

10. Sprague AH, Khalil RA (2009): Inflammatory cytokines in vascular dysfunction and vascular disease. Biochem Pharmacol 78: 539-552.

11. Gill SE, Parks WC (2008): Metalloproteinases and their inhibitors: regulators of wound healing. Int J Biochem Cell Biol 40: 1334-1347.

12. Basu R, Lee J, Morton JS, et al. (2013): TIMP3 is the primary TIMP to regulate agonist-induced vascular remodelling and hypertension. Cardiovasc Res 98: 360-371.

13. Schultz GS, Sibbald RG, Falanga V, et al. (2003): Wound bed preparation: a systematic approach to wound management. Wound Repair Regen 11 Suppl 1: S1-S28.

14. Serra R, Buffone G, Falcone D, et al. (2013): Chronic venous leg ulcers are associated with high levels of metalloproteinases-9 and neutrophil gelatinase-associated lipocalin. Wound Repair Regen 21: 395-401.

15. Grzela T, Niderla-Bielinska J, Litwiniuk M, White R. (2014): The direct inhibition of MMP-2 and MMP-9 by an enzyme alginogel: a possible mechanism of healing support for venous leg ulcers. J Wound Care 23: 278-285.

16. Parish LC, Dryjski M, Cadden S; Versiva XC Pressure U1cer Study Group (2008): Prospective clinical study of a new adhesive gelling foam dressing in pressure ulcers. Int Wound J 5: 60-67.

17. Philbin S (2013): Pressure ulcer management using sodium carboxymethylcellulose hydrofiber® foam dressings. Ostomy Wound Manage 59: 10-12.

18. Amato B, Coretti G, Compagna R, et al. (2013): Role of matrix metalloproteinases in non-healing venous ulcers. Int Wound $\mathrm{J}$ [Epub ahead of print].

19. Ladwig GP, Robson MC, Liu R, et al. (2002): Ratios of activated matrix metalloproteinase- 9 to tissue inhibitor of matrix metalloproteinase-1 in wound fluids are inversely correlated with healing of pressure ulcers. Wound Repair Regen 10: 26-37.
20. Litwiniuk M, Bikowska B, Niderla-Bielińska J, et al. (2012): Potential role of metalloproteinase inhibitors from radiation-sterilized amnion dressings in the healing of venous leg ulcers. Mol Med Rep 6: 723-728.

21. Rayment EA, Upton Z, Shooter GK (2008): Increased matrix metalloproteinase-9 (MMP-9) activity observed in chronic wound fluid is related to the clinical severity of the ulcer. $\mathrm{Br}$ J Dermatol 158: 951-961.

22. Wiegand C, White RJ (2013): Binding and inhibition of protease enzymes, including MMPs, by a superabsorbent dressing in vitro. J Wound Care 22: 221-227. 